\title{
Photo-switching spin pairs-synergy between LIESST effect and magnetic interaction in an iron(II) binuclear spin-crossover compound $\dagger$
}

\author{
Guillaume Chastanet, ${ }^{a}$ Ana B. Gaspar, ${ }^{b}$ José Antonio Real ${ }^{* b}$ and Jean-François Létard ${ }^{* a}$ \\ a Institut de Chimie de la Matière Condensée de Bordeaux, Laboratoire des Sciences Moléculaires, UPR \\ CNRS No. 9048, 87 Av. du Doc. A. Schweitzer, 33608 Pessac, France. \\ E-mail:letard@chimsol.icmcb.u-bordeaux.fr \\ ${ }^{b}$ Departament de Química Inorgánica/Institut de Ciencia Molecular, Universitat de València, Dr. \\ Moliner 50, 46100 Burjassot, València, Spain
}

\section{Received (in Cambridge, UK) 10th January 2001, Accepted 22nd March 2001 First published as an Advance Article on the web 6th April 2001}

\begin{abstract}
The decrease of the magnetic response under irradiation at very low temperature was interpreted as a new evidence of synergy between magnetic interaction and spin transition in an iron(II) binuclear SC compound.
\end{abstract}

The design and the synthesis of polynuclear complexes with versatile chemical and physical properties are of current interest in the areas of molecule-based electronic, photochemistry and magnetism. The goal is to develop novel materials whose properties can be controlled by the input of external information. In this context, particular interest has been focused on iron(II) spin-crossover (SC) compounds because they can be considered among the best examples of switchable coordination molecules. A reversible transition between the paramagnetic high-spin state (HS, $S=2$ ) and the diamagnetic low-spin state (LS, $S=0$ ) can be induced by a change in temperature, pressure or by light irradiation. ${ }^{1}$ This latter effect opens some interesting perspectives to design optical switches. In 1984, Decurtins et al. discovered that by irradiation with green light the SC compound $\left[\mathrm{Fe}(\mathrm{ptz})_{6}\right]\left(\mathrm{BF}_{4}\right)_{2}(\mathrm{ptz}=1$-propyltetrazole $)$ at $20 \mathrm{~K}$ could be converted from the stable LS state to the metastable HS state. ${ }^{2}$ Later, Hauser reported the reverse-LIESST effect, wherein red light is used to convert back the compound into the LS state. ${ }^{3}$

Up to now, most of the SC compounds exhibiting LIESST properties are assemblies of monomeric units with throughspace rather than through-bond interactions. Allowing SC centers to communicate by chemical bridges, i.e. oligomeric or polymeric species, is an important strategy to explore and enhance cooperativity of a given system. ${ }^{4,5}$ Along this line, binuclear compounds of formula $\left[\left\{\mathrm{Fe}(\mathrm{L})(\mathrm{NCX})_{2}\right\}_{2}(\mathrm{bpym})\right](\mathrm{L}$ $=$ bpym $\left(2,2^{\prime}\right.$-bipyrimidine $)$, bt $\left(2,2^{\prime}\right.$-bithiazoline $)$ and $\mathrm{X}=\mathrm{S}$, $\mathrm{Se})$ represent one of the most unusual family in the SC field. These compounds are the simplest example of polynuclear SC complexes exhibiting a rich variety of magnetic regimes. Further, they are the only iron(II) example where the interplay between both spin crossover and intramolecular magnetic exchange phenomena are present in the same molecule. In $\left[\left\{\mathrm{Fe}(\text { bpym })(\mathrm{NCS})_{2}\right\}_{2}\right.$ (bpym) $]$ the two iron(II) ions are in the HS state and couple antiferromagnetically through the bridge bpym $\left(J=-4.1 \mathrm{~cm}^{-1}\right.$ in the zero-field Hamiltonian $\left.H=-J S_{\mathrm{A}} S_{\mathrm{B}}\right)$ giving an $S=0$ ground pair state HS-HS at low temperature. ${ }^{6}$ In contrast, $\left[\left\{\mathrm{Fe}(\text { bpym })(\mathrm{NCSe})_{2}\right\}_{2}\right.$ (bpym) $]$ shows a one-step transition involving $50 \%$ of iron atoms at $T_{\mathrm{c}}=125 \mathrm{~K},{ }^{7}$ whereas an almost complete two-step spin transition in the range $160-210 \mathrm{~K}$ takes place for $\left[\left\{\mathrm{Fe}(\mathrm{bt})(\mathrm{NCS})_{2}\right\}_{2}(\mathrm{bpym})\right] .{ }^{8}$ This behavior was interpreted in terms of the occurrence of LS-LS $\leftrightarrow$ LS-HS $\leftrightarrow$ HS-HS pair spin states in each binuclear unit. ${ }^{8}$ The stabilisation of the LS-HS mixed spin pair was assigned to the synergy effect between anticooperative intramolecular and cooperative intermolecular interactions.

$\dagger$ Dedicated to the memory of Olivier Kahn.
Recently, we have reported the unusual photomagnetic behavior stemming from the interplay between spin-crossover and magnetic coupling phenomena. ${ }^{9}$ At low temperature, light switches [ $\left.\left\{\mathrm{Fe}(\mathrm{bt})(\mathrm{NCS})_{2}\right\}_{2}(\mathrm{bpym})\right]$ from the $S=0$ spin state of the LS-LS pair to the $S=0$ spin state of the HS-HS pair. Warming the sample first reveals the latent magnetic coupling between the HS iron(II) ions as the $S=1,2,3$ and 4 excited states are populated, then switches off this virtual pair spin state at temperatures as high as $60 \mathrm{~K}$ where molecules have enough energy to overcome the barrier associated with the change of molecular geometry upon spin conversion. As a continuation of this study we have investigated the LIESST effect on $[\{\mathrm{Fe}-$ $($ bpym $\left.)(\mathrm{NCSe})_{2}\right\}_{2}$ (bpym)]. As mentioned above, this compound exhibits an incomplete thermal spin transition interpreted in terms of HS-HS $\leftrightarrow \mathrm{LS}-\mathrm{HS}$ conversion at $125 \mathrm{~K}$ (Fig. 1). The slight decrease of the magnetic response as $T$ approaches absolute zero is due to zero-field splitting of the $S=2$ spin state of the mixed-spin pair.

At $10 \mathrm{~K}$, we observed that green light irradiation $(530.9 \mathrm{~nm}$, power $\left.10 \mathrm{~mW} \mathrm{~cm}^{-2}\right)^{10}$ induces a decrease of $\chi_{\mathrm{M}} T$ from 2.6 to $1.6 \mathrm{~cm}^{3} \mathrm{~K} \mathrm{~mol}^{-1}$. Similar behavior was recorded at 647-679, $759-799$ and $830 \mathrm{~nm}$. Usually, the observation of a decrease in the magnetic response under light irradiation is a signature of reverse-LIESST as the HS state is converted into the LS state. However, for $\left[\left\{\mathrm{Fe}(\mathrm{bpym})(\mathrm{NCSe})_{2}\right\}_{2}(\mathrm{bpym})\right]$ this situation is no longer valid because of the occurrence of the unusual HS-LS state and the virtual excited HS-HS state which involves a latent antiferromagnetic interaction similar in magnitude as observed for $\left[\left\{\mathrm{Fe}(\mathrm{bt})(\mathrm{NCS})_{2}\right\}_{2}(\mathrm{bpym})\right] .{ }^{9}$ Hence, a decrease of the magnetic response should be expected upon irradiation.

Fig. 1 compares $\chi_{\mathrm{M}} T$ vs. $T\left(\chi_{\mathrm{M}}\right.$ is the molar magnetic susceptibility and $T$ the temperature) of the photoinduced

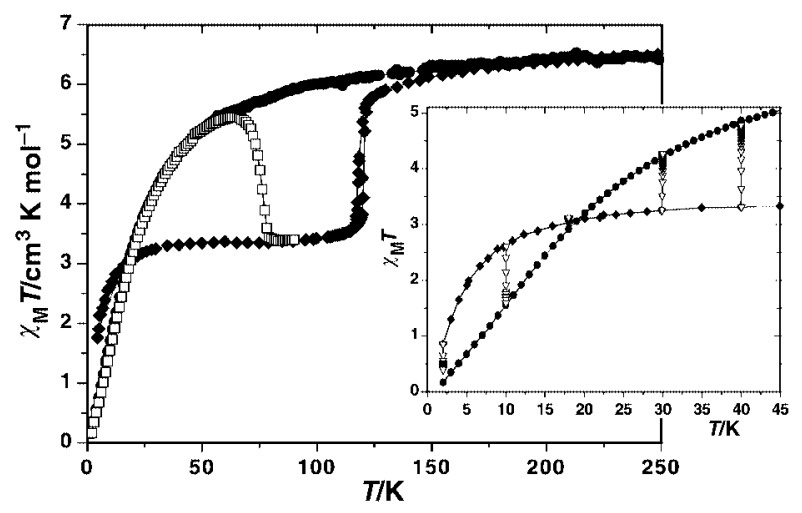

Fig. $1 \chi_{\mathrm{M}} T$ vs. $T$ for $(\diamond)\left[\left\{\mathrm{Fe}(\mathrm{bpym})(\mathrm{NCSe})_{2}\right\}_{2}(\mathrm{bpym})\right]$ and $(\mathbf{O})$ $\left[\left\{\mathrm{Fe}(\mathrm{bpym})(\mathrm{NCS})_{2}\right\}_{2}(\mathrm{bpym})\right]$. ( $\left.\square\right)$ refers to the warming mode of $\left[\left\{\mathrm{Fe}(\text { bpym })(\mathrm{NCSe})_{2}\right\}_{2}\right.$ (bpym) $]$ after the irradiation was applied for $1 \mathrm{~h}$ at 10 $\mathrm{K}$, then turned off. Inset: data obtained with irradiation $(\nabla)$ for $1 \mathrm{~h}$ at 2,10 , 17,30 and $40 \mathrm{~K}$ 


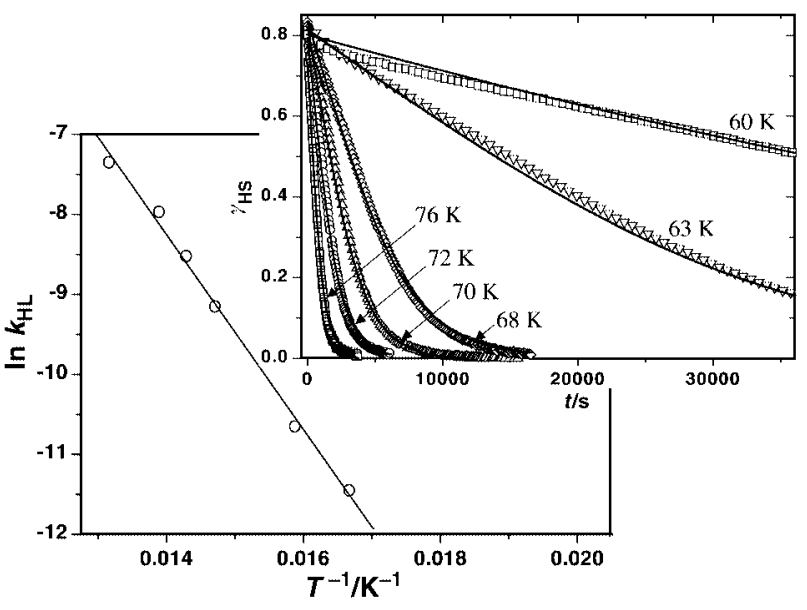

Fig. 2 Ln $k_{\mathrm{HL}}$ vs. $1 / T$ curve of [ $\left\{\mathrm{Fe}(\mathrm{bpym})(\mathrm{NCSe})_{2}\right\}_{2}$ (bpym)]. Inset: time dependence at various temperatures of the HS molar fraction generated by light irradiation at $10 \mathrm{~K}$.

$\left[\left\{\mathrm{Fe}(\text { bpym })(\mathrm{NCSe})_{2}\right\}_{2}(\right.$ bpym $\left.)\right]$ with the binuclear $[\{\mathrm{Fe}-$ (bpym)(NCS $\left.)_{2}\right\}_{2}$ (bpym)] compound containing two iron(II) ions in the HS-HS state in the whole temperature range. ${ }^{6-8}$ Both magnetic responses are similar below $40 \mathrm{~K}$. It is well known that the interaction parameter, $J$, in a coupled binuclear compound depends essentially on the nature of the chemical bridge. As for both compounds the bridging network is similar, in case of a quasi-quantitative LS-HS $\rightarrow$ HS-HS conversion (LIESST effect), the magnetic properties of $\left[\left\{\mathrm{Fe}(\mathrm{bpym})(\mathrm{NCSe})_{2}\right\}_{2^{-}}\right.$ (bpym) $]$ after irradiation and $\left[\left\{\mathrm{Fe}(\text { bpym })(\mathrm{NCS})_{2}\right\}_{2}(\right.$ bpym $\left.)\right]$ should be very similar. In contrast, a LS-LS population (reverse-LIESST) should lead to a diamagnetic ground state for $\left[\left\{\mathrm{Fe}(\mathrm{bpym})(\mathrm{NCSe})_{2}\right\}_{2}(\mathrm{bpym})\right]$ very different from the magnetic properties of $\left[\left\{\mathrm{Fe}(\mathrm{bpym})(\mathrm{NCS})_{2}\right\}_{2}(\mathrm{bpym})\right]$. The data displayed on Fig. 1 are in favor of the first alternative.

Fig. 1 also shows the $\chi_{\mathrm{M}} T$ value of $\left[\left\{\mathrm{Fe}(\mathrm{bpym})(\mathrm{NCSe})_{2}\right\}_{2^{-}}\right.$ (bpym)] under light irradiation at 2, 10, 17, 30 and $40 \mathrm{~K}$. In all cases, the photostationary point reached the $\chi_{\mathrm{M}} T$ limit of $\left[\left\{\mathrm{Fe}(\text { bpym })(\mathrm{NCS})_{2}\right\}_{2}\right.$ (bpym)], confirming the HS-HS population. ${ }^{11}$ What is remarkable is that at $17 \mathrm{~K}$, both before and after irradiation, the compound has a similar magnetic response. This does not mean that the LIESST effect does not occur. In fact, we are faced with two totally different spin states. Before irradiation, the system acts as a HS-LS mixed-spin pair, resulting in a $S=2$ spin state with a $\chi_{\mathrm{M}} T$ value of $3 \mathrm{~cm}^{3} \mathrm{~K}$ $\mathrm{mol}^{-1}$. After irradiation, the two iron(II) are HS and coupled antiferromagnetically with a thermal population of the lowlying spin states, $S=1-4$, located at energies $E(S)=-J S(S+$ $1) / 2$ above the ground state $S=0$. At $17 \mathrm{~K}$, the population of the HS-HS pair, assuming a $J$ factor of $-4.1 \mathrm{~cm}^{-1}$ and a $g$ value of 2.13 , is $16 \% S=0,35 \% S=1,29 \% S=2,15 \% S=3$ and $5 \%$ $S=4$. The resulting $\chi_{\mathrm{M}} T$ value is then $3 \mathrm{~cm}^{3} \mathrm{~K} \mathrm{~mol}^{-1}$ and similar to that of the HS-LS mixed-spin pair.

The kinetics of the relaxation were investigated from $2 \mathrm{~K}$ up to $75 \mathrm{~K}$ (Fig. 2). Below $55 \mathrm{~K}$, the relaxation is very slow, for instance, at $10 \mathrm{~K}$ the magnetic response varies by $\mathrm{ca} .0 .01 \%$ in $13 \mathrm{~h}$, which requires relaxation rates smaller than $10^{-6} \mathrm{~s}^{-1}$. From 55 to $75 \mathrm{~K}$, the dynamic of the relaxation is faster and falls within the time window of our set-up. This behavior matches the theory of HS $\rightarrow$ LS relaxation proposed by Buhks et al. ${ }^{12}$ which predicts a temperature-independent relaxation rate, i.e. a tunneling process, at low temperature and an activation relaxation process at higher temperatures. The decays of the HS molar fraction, $\gamma_{\mathrm{HS}}, v s$. time, at various temperatures, are also represented in Fig. $2 ; \gamma_{\mathrm{HS}}$ is deduced from $\left[\left(\chi_{\mathrm{M}} T\right)_{h v}-\right.$ $\left.\left(\chi_{\mathrm{M}} T\right)_{\mathrm{HS}-\mathrm{LS}}\right] /\left[\left(\chi_{\mathrm{M}} T\right)_{\mathrm{AF}}-\left(\chi_{\mathrm{M}} T\right)_{\mathrm{HS}-\mathrm{LS}}\right] \cdot{ }^{13}$ An analysis of these data indicates that the relaxation curves can be satisfactorily fitted by a sigmoidal law. ${ }^{14}$ This reflects a self-acceleration process as $\gamma_{\mathrm{HS}}$ decreases. The plot of $\ln k_{\mathrm{HL}} v s .1 / T$ (Fig. 2) gives a straight line, with an activation energy $E_{\mathrm{a}}=850 \mathrm{~cm}^{-1}$, a preexponential factor $k_{\infty}=6.6 \times 10^{3} \mathrm{~s}^{-1}$ and an additional activation energy associated to the cooperativity $E_{\mathrm{a}} *=53$ $\mathrm{cm}^{-1}$. Such parameters allow understanding of the shape of the $T_{\mathrm{c}}$ (LIESST) experiment ${ }^{14}$ reported in Fig. 1 . In the tunneling region where the kinetic of the relaxation back to the HS-LS state is very slow, the $S=1-4$ spin states of the local HS-HS pair are progressively populated and $\chi_{\mathrm{M}} T$ increases. Above 55 $\mathrm{K}$, the relaxation process of the local HS-HS pair becomes thermally activated and a deviation appears with the magnetic properties of $\left[\left\{\mathrm{Fe}(\mathrm{bpym})(\mathrm{NCS})_{2}\right\}_{2}(\mathrm{bpym})\right]$. The critical $T_{\mathrm{c}}$ (LIESST) temperature is found at $75 \mathrm{~K}$.

In summary, this communication reports a new example of synergy between magnetic interaction and spin transition under light irradiation in an iron(II) binuclear SC compound. For the first time at very low temperature, the photoconversion of a LSHS into a HS-HS pair, according to the LIESST effect, results in a decrease in the magnetic response. This behavior in the complex $\left[\left\{\mathrm{Fe}(\text { bpym })(\mathrm{NCSe})_{2}\right\}_{2}\right.$ (bpym) $]$ arises from the conversion of the $S=2$ initial state into an $S=0$ low-lying spin state by light irradiation.

We are grateful for financial assistance from the European Commission for granting the TMR-Network 'Thermal and Optical Switching of Spin States (TOSS)', Contract No. ERBFMRX-CT98-0199. Also we would like to thank the financial assistance of the Spanish DGICYT through Project PB97-1397 and of the PICASSO program.

\section{Notes and references}

1 P. Gütlich, A. Hauser and H. Spiering, Angew. Chem., Int. Ed. Engl., 1994, 33, 2024.

2 S. Decurtins, P. Gütlich, C. P. Köhler, H. Spiering and A. Hauser, Chem. Phys. Lett., 1984, 105, 1.

3 A. Hauser, Chem. Phys. Lett., 1986, 124, 543.

4 O. Kahn and C. Jay Martinez, Science, 1998, 279, 44.

5 J. A. Real, E. Andrés, M. Carmen Muñoz, M. Julve, T. Granier, A. Bousseksou and F. Varret, Science, 1995, 268, 265.

6 J. A. Real, I. Castro, A. Bousseksou, M. Verdaguer, R. Burriel, M. Castro, J. Linares and F. Varret, Inorg. Chem., 1997, 36, 455.

7 J. A. Real, J. Zarembowitch, O. Kahn and X. Solans, Inorg. Chem., 1987, 26, 2939.

8 J. A. Real, H. Bolvin, A. Bousseksou, A. Dworkin, O. Kahn, F. Varret and J. Zarembowitch, J. Am. Chem. Soc., 1992, 114, 4650.

9 J.-F. Létard, J. A. Real, N. Moliner, A. B. Gaspar, L. Capes, O. Cador and O. Kahn, J. Am. Chem. Soc., 1999, 121, 10630.

10 Irradiation was carried out by using a $\mathrm{Kr}^{+}$laser or a diode laser $(830 \pm$ $15 \mathrm{~nm}$ ). The measurements were performed on a very thin layer of powder sample. The weight was estimated by comparing the thermal SC curve with the curve recorded with an heavier and accurately weighed sample.

11 Result in agreement with applied-field Mössbauer spectroscopy used to detect coupled states of iron(II). V. Ksenofontov, H. Spiering, A. B. Gaspar, J. A. Real and P. Gütlick, International Conference on Molecular Magnets, San Antonio, TX, September 16-21, 2000.

12 E. Buhks, G. Navon, M. Bixon and J. Jortner, J. Am. Chem. Soc., 1980, 102, 2918.

$13\left(\chi_{\mathrm{M}} T\right)_{h v}$ represents the magnetic response reached after irradiation, $\left(\chi_{\mathrm{M}} T\right)_{\mathrm{HS}-\mathrm{LS}}$ corresponds to the HS-LS mixed-spin pair and $\left(\chi_{\mathrm{M}} T\right)_{\mathrm{AF}}$ is calculated according to the Van Vleck equation with the zero-field spin Hamiltonian being expressed as $H=-J S_{\mathrm{A}} \cdot S_{\mathrm{B}}$; see O. Kahn, Molecular Magnetism, VCH, New York, 1993.

14 In the thermally activated domain the relaxation rate is given by $\partial \gamma_{\mathrm{LS}} / \partial t$ $=-k_{\mathrm{HL}}^{*} \gamma_{\mathrm{LS}}$ with $k_{\mathrm{HL}}^{*}=k_{\mathrm{HL}} \exp \left(-\left(E_{\mathrm{a}}^{*} / k T\right) \gamma_{\mathrm{LS}}\right)$ and $k_{\mathrm{HL}}=k_{\infty} \exp$ $\left(-\left(E_{\mathrm{a}} / k T\right)\right)$; see A. Hauser, Chem. Phys. Lett., 1992, 192, 65.

$15 T_{\mathrm{c}}$ (LIESST) refers to the limit temperature where, in a SQUID magnetometer, the photoinduced HS information is erased. The procedure is reported in: J.-F. Létard, O. Capes, G. Chastanet, N. Moliner, S. Létard, J. A. Real and O. Kahn, Chem. Phys. Lett., 1999, 313, 115 . 